\title{
Do Pain and Function Improve after Dextrose Prolotherapy or Autologous Platelet-Rich Plasma Injection in Longstanding Groin Pain?
}

\author{
Omer Ozkan', Serife Seyma Torgutalp', Levend Karacoban', Gurhan Donmez', Feza \\ Korkusuz ${ }^{1}$
}

Affiliations: 'Hacettepe University, Faculty of Medicine, Department of Sports Medicine, Ankara, Turkey

Correspondence: O. Ozkan, Hacettepe University, Faculty of Medicine, Department of Sports Medicine, 06100, Ankara, Turkey. E-mail: dr.omerozkan@hotmail.com

\begin{abstract}
Longstanding groin pain is a non-infectious and inflammatory chronic condition that causes inguinal pain in athletes. We compared dextrose prolotherapy (15\% dextrose solution) and platelet-rich plasma (autologous platelet gel, plasma rich in growth factors, platelet concentrate) injections in male soccer players with longstanding groin pain. Forty elite male soccer players, ages between 13 and 33, with longstanding groin pain were treated. Patients were randomly divided into the dextrose prolotherapy $(n=9)$ and platelet-rich plasma injection $(n=6)$ groups. Following three injections, all patients were enrolled in the 12-week progressive home exercise protocol. Pain and function were assessed using the visual analogue (VAS) and Nirschl Pain Phase Scale (NPPS) scores before, one month after, and six months after the injection, respectively. VAS and NPPS scores decreased in both of the dextrose prolotherapy and the platelet-rich plasma injection groups. A decrease in pain and improvement in function were evident at a month after injection, and they remained favourable at the end of six months (VAS overall, $p<0.001$ and $p=0.003$, respectively) (NPPS overall, $p<0.001$ and $p=0.007$, respectively). A difference between the dextrose prolotherapy and the platelet-rich plasma injection groups was not observed at both time points (VAS, $p=0.3, p=0.7$, and $p=0.6$ respectively) (NPPS, $\mathrm{p}=0.6, \mathrm{p}=0.9$, and $\mathrm{p}=0.9$ respectively). Dextrose prolotherapy and platelet-rich plasma injections decreased pain and improved function in longstanding groin pain treatment of male soccer players. These treatments should be considered in patients who are not responding to conservative treatment modalities.
\end{abstract}

KEY WORDS longstanding groin pain, athletic pubalgia, injection, symphysis pubis, bone marrow oedema

$@$ MJSSMontenegro

DEXTROSE PROLOTHERAPY FOR LONGSTANDING GROIN PAIN

http://mjssm.me/?sekcija=article\&artid=196

\section{Introduction}

Longstanding groin pain is a non-infectious and inflammatory chronic condition that affects symphysis pubis and surrounding tissues (Jardí et al., 2014). It is an overuse injury of the groin area in athletes participating in strenuous athletic activities, especially among soccer players (Angoules, 2015). Kicking, turning, twisting, cutting, pivoting, and sprinting activities associated with sports such as soccer, rugby, gridiron football, ice hockey, Australian rules football, and distance running have been linked to this pathology (Angoules, 2015; Jardí et al., 2014). The incidence rate of groin pain is estimated at $10 \%$ to $18 \%$ per year among male soccer players (Hölmich et al., 1999). Groin pain is often accompanied by other adductor or hip pathologies (Omar et al., 2008), such as a microtear of the adductor longus tendon attachment at the pubis, rather than an isolated entity and time to complete resolution of symptoms may extend up to 1 year (Cunningham et al., 2007). Failure to return to the prior level of sports function may be as high as 25\% (Batt, McShane, \& Dillingham, 1995).

Different treatments have been recommended for longstanding groin pain, ranging from conservative management to surgical procedures (Choi, McCartney, \& Best, 2011; McKim, Taunto, \& Kirchner, 1999; Scarpone, Rabago, Zgierska, Arbogast, \& Snell, 2008; Scholten, Massimi, Dahmen, Diamond, \& Wyss, 2015; Topol, Reeves, \& Hassanein, 2005; Watkin, Gallegos, Moisey, \& Charlton, 1995). Conservative treatment initially in-

Received: September 222019 | Accepted after revision: December 152019 | First published online: Septembar 012020

(c) 2020 by the author(s). License MSA, Podgorica, Montenegro. This article is an open access article distributed under the terms and conditions of the Creative Commons Attribution (CC BY).

Conflict of interest: None declared. 
cludes rest, limited activity, ice and use of anti-inflammatory medication, which is followed by a rehabilitation program (Hopp, Culemann, Kelm, Pohlemann, \& Pizanis, 2013). Other suggested treatment options include steroids and local anaesthetic (Choi et al., 2011), dextrose prolotherapy (DP) (Topol et al., 2005), platelet-rich plasma (PRP) (Scholten et al., 2015) injections, anticoagulation therapy (Watkin et al., 1995), and compression shorts (McKim et al., 1999).

Dextrose prolotherapy, which consists of concentrated dextrose as an irritant, has been used in ligament or tendon insertions as a proliferant (Scarpone et al., 2008). The proliferative response to dextrose was thought to be a result of the higher osmolarity of the injected solution than that of the interstitial tissue. Evidence suggests the release of transforming growth factor beta-1, platelet-derived growth factor, connective tissue growth factor, epithelial growth factor, and basic fibroblastic growth factor were stimulated when exposed to various glucose concentrations (Murphy et al., 1999). DP has been reported to decrease pain and improve function in the treatment of musculoskeletal pain and sports-related soft tissue injuries (Scarpone et al., 2008). PRP injection is a relatively newer method than DP injection (Scholten et al., 2015). With PRP injection, high concentrations of growth factors are transmitted directly to a lesion, which in turn initiates the natural healing process (Scholten et al., 2015).

A few studies with an encouraging biological basis and theory on DP and PRP injection for groin pain have suggested the beneficial effects of these treatments (Scholten et al., 2015; Topol et al., 2005). However, applicable data are insufficient to support the routine clinical use of these therapies in this disease. To our knowledge, no previous study has compared the effects of DP and PRP injection in the treatment of groin pain. This study thus investigated whether DP and PRP injections could decrease pain and improve the function of longstanding groin pain in elite male soccer players. Our aim was to compare the effects of DP and PRP injections on pain and function at one and six months using the visual analogue scale (VAS) and the Nirschl Pain Phase Scale (NPPS), respectively.

\section{Methods}

Patients

A retrospective cohort study in patients with longstanding groin pain was designed. Data of 15 elite male soccer players with the diagnosis of longstanding groin pain who were referred to the Sports Medicine Department were analysed. The Hacettepe University Ethics Committee on Research with Human Subjects (Decision Number: GO 18/1205) approved the study. The median age of all patients was 20 years old (min: 18 years old, max: 33 years old). There was no significant difference in age between the DP and the PRP groups ( $\mathrm{p}=0.07)$ (Table 1).

\begin{tabular}{|c|c|c|c|}
\hline & $\begin{array}{l}\text { DP group } \\
(n=9)\end{array}$ & $\begin{array}{l}\text { PRP group } \\
(n=6)\end{array}$ & $\mathbf{P}$ \\
\hline Age, years old, median (min-max) & $19(18-33)$ & $22.5(20-32)$ & 0.07 \\
\hline \multicolumn{4}{|l|}{ VAS scores, median (min-max) } \\
\hline Pre-injection & $8(5-9)$ & $7(5-9)$ & 0.4 \\
\hline $1^{\text {st }}$ months & $2(1-3)$ & $2(1-6)$ & 0.8 \\
\hline $6^{\text {th }}$ months & $1(0-2)$ & $1(0-6)$ & 0.7 \\
\hline \multicolumn{4}{|l|}{ NPPS scores, median (min-max) } \\
\hline Pre-injection & $5(3-7)$ & $4.5(3-6)$ & 0.6 \\
\hline $1^{\text {st }}$ months & $2(1-2)$ & $1.5(1-5)$ & 0.9 \\
\hline $6^{\text {th }}$ months & $1(0-1)$ & $0.5(0-5)$ & 0.9 \\
\hline
\end{tabular}

Note. DP - dextrose prolotherapy, min - minimum, max - maximum, NPPS - Nirschl Pain Phase Scale, PRP - platelet-rich plasma.

Patients with anterior and medial groin pain for at least six months, who resisted to non-steroidal anti-inflammatory medicine (NSAIDs), stretching, physical therapy modalities and other conservative treatment methods, were included. Groin pain was defined as pain that is exacerbated by walking, pelvic motion, adductor stretching, abdominal muscle strengthening exercises, or movement from a seated to a standing position, and may radiate into the lower abdominal muscles, perineum, inguinal region, scrotum or medial thigh. All of the participants had pain on the pubic symphysis and the pubic ramus. The diagnosis of longstanding groin pain was made by clinical examination that included tenderness on palpation of the symphyseal region, the Single Adductor, the Adductor Squeeze, and the Bilateral Adductor tests (Verrall, Slavotinek, Barnes, \& Fon, 2005).

Clinical staging was based on the classification proposed by Rodriguez et al. (2001) that included clinical findings and diagnostic features. All of the patients clinical stages were Stage III (bilateral symptoms, inguinal pain involving the adductor and abdominal muscles, pain with kicking, sprinting, directional changes, etc., inability to continue sport participation) or Stage IV (inguinal pain involving the adductor and abdominal muscles, pain re- 
ferred to the pelvic girdle and lumbar spine with defecation, sneezing, and walking on uneven terrain, inability to perform activities of daily living). Longstanding groin pain diagnosis was confirmed with magnetic resonance imaging (MRI) in each soccer player, which is the standard technique for longstanding groin pain. A hyper-intense signal on T2-weighted images within the symphysis and adjacent parasymphyseal region and bilateral symphyseal subchondral bone marrow oedema extending from anterior to posterior were present in all patients' MRI.

Patients were excluded from the study if they had received local steroid injections within the previous six months or NSAIDs within the first week before their clinical application. Patients with a sports hernia, inguinal wall deficiency, iliopsoas strain, stress, and avulsion fractures, intra-articular hip joint injury or other relevant pathologies, such as snapping hip syndrome, low back pain, and nerve compression, were excluded.

\section{Treatment procedures}

Dextrose Prolotherapy Preparation and Injection

Dextrose prolotherapy was prepared by mixing $6 \mathrm{~mL}$ of $20 \%$ dextrose solution (Polifleks, Polifarma, Turkey) and $2 \mathrm{~mL}$ of prilocaine (Citanest, AstraZeneca, UK), resulting in an $8 \mathrm{~mL} 15 \%$ solution. The injection area was prepared with povidone-iodine (Isosol, Merkez, Turkey), to prevent infection. After this preparation, the dextrose solution was injected using a 22G needle (BD Microlance, Becton, Dickinson and Company, NJ, USA) into the tenderest pubic area using the peppering technique (a single skin portal and 3 or 4 penetrations to the tendon fascia), while the patient was in a supine position with a leg slightly abducted and externally rotated at the hip. Three DP injections were performed at one-week intervals. Patients were told not to exercise during the three weeks of the injection period.

\section{PRP Preparation and Injection}

Fifty millilitres $(50 \mathrm{ml})$ of blood was collected from the veins in antecubital fossa into sodium citrate tubes (BD Vacutainer sodium citrate tubes, Becton, Dickinson and Company, NJ, USA). The blood was then centrifuged for eight (8) minutes at $1500 \mathrm{rpm}$ using a desktop centrifuge (NF 800, Nüve, Turkey). This spin separated the whole blood into three layers: an upper layer consisting mainly of plasma and platelets, a middle layer consisting of white blood cells (known as the buffy coat), and a bottom layer consisting mainly of red blood cells (Dhurat \& Sukesh, 2014). The upper layer with the buffy coat was transferred to sterile tubes, and a second centrifugation was utilized to concentrate platelets. The upper two thirds of the plasma were platelet-poor plasma, while the lower third was PRP. After extraction of the platelet-poor plasma, $5 \mathrm{ml}$ of PRP was attained. This was injected using a 22G needle (BD Microlance 3, Becton, Dickinson and Company, NJ, USA) into the most tender pubic area using the peppering technique as described for DP.

\section{Rehabilitation Program after Injection}

Systemic and local complications related to injections were not observed in both groups. After the last injection, patients received the same 12 -week progressive home exercise protocol including a range of motion, core stability, stretching, cardiovascular training, strengthening exercises of the pelvis, hip, abdominal and gluteal muscles by a sports physician.

Patients were told not to run during the first week after injection. From Days 7 to 14 they could jog, and from Days 14 to 28 they were able to increase distance and speed as tolerated. Forceful kicking was avoided for 28 days. Patients were not allowed to take NSAIDs or any other painkillers during this period.

Follow-up data

Data were obtained on all patients before, one month after, and six months after the first injection. Outcomes were measured through VAS scores for pain during exercise or sport, and NPPS scores of athletic overuse injuries, which has seven phases of disability based on injury severity, to determine the functional impairment level (Table 2) (O’Connor, Howard, Fieseler, \& Nirschl, 1997).

TABLE 2. Nirschl Pain Phase Scale of Athletic Overuse Injuries (O'Connor et al., 1997)

\begin{tabular}{|c|c|}
\hline Phase & Description \\
\hline Phase 1 & Stiffness or mild soreness after activity. Pain is usually gone within 24 hours. \\
\hline Phase 2 & $\begin{array}{l}\text { Stiffness or mild soreness before activity that is relieved by warm-up. Symptoms are not present } \\
\text { during activity but return afterward, lasting up to } 48 \text { hours. }\end{array}$ \\
\hline Phase 3 & $\begin{array}{l}\text { Stiffness or mild soreness before specific sport or occupational activity. Pain is partially relieved by } \\
\text { warm-up. It is minimally present during activity, but does not cause the athlete to alter activity. }\end{array}$ \\
\hline Phase 4 & $\begin{array}{l}\text { Similar to phase } 3 \text { pain but more intense, causing the athlete to alter performance of the activity. } \\
\text { Mild pain occurs with activities of daily living but does not cause a major change in them }\end{array}$ \\
\hline Phase 5 & $\begin{array}{l}\text { Significant (moderate or greater) pain before, during, and after activity, causing alteration of activity. } \\
\text { Pain occurs with activities of daily living but does not cause a major change in them. }\end{array}$ \\
\hline Phase 6 & $\begin{array}{l}\text { Phase } 5 \text { pain that persists even with complete rest. Pain disrupts simple activities of daily living and } \\
\text { prohibits doing household chores. }\end{array}$ \\
\hline Phase 7 & Phase 6 pain that also disrupts sleep consistently. Pain is aching in nature and intensifies with activity. \\
\hline
\end{tabular}




\section{Statistical Analyses}

The type of injections, PRP or DP, was the independent variable. VAS and NPPS scores were dependent variables. All statistical analyses were performed using IBM SPSS Statistics for Windows, V22.0 (IBM Corp). Variables were assessed using the visual (histograms, probability plots) and analytical methods (Kolmogorov-Smirnov/Shapiro-Wilk's test) for normal distribution. Descriptive analyses were presented using frequencies, medians, and minimum-maximum values since variables were not normally distributed. The Mann-Whitney U test was used to compare age, VAS, and NPPS scores between DP and PRP groups. Friedman tests were conducted to whether there is a significant change in the VAS and NPPS scores over time. The Wilcoxon test was performed to test the significance of pairwise differences using the Bonferroni correction to adjust for multiple comparisons; $5 \%$ type-I error level was used to infer statistical significance.

\section{Results}

Visual Analogue Scale Scores

A decrease in pain was observed in a month according to VAS within each group compared to the initial scores (Table 1). Both groups showed significant improvements in the VAS scores at all follow-ups. There were no significant differences between groups regarding VAS scores at pre-injection, 1st month and 6th month of follow-up ( $\mathrm{p}=0.3, \mathrm{p}=0.7$, and $\mathrm{p}=0.6$ respectively) (Figure 1$)$. In the DP group, the median VAS scores were 8 (min: 5, max: 9) at pre-injection, 2 (min: 1, max: 3) at first month and 1 (min: 0, max: 2 ) at sixth month, and the decrease of VAS through time points was statistically significant (overall $\mathrm{p}<0.001$, from pre-injection to 1 st month $p=0.007$, from 1 st month to 6 th month $p=0.006$ ). In the PRP group, the median VAS scores were 7 (min: 5, max: 9) at pre-injection, 2 (min: 1, max: 6) at first month and 1 (min: 0, max: 6) at sixth month, and the decrease of VAS through time points was statistically significant (overall $\mathrm{p}=0.003$, from pre-injection to 1 st month $\mathrm{p}=0.027$, from 1 st month to 6 th month $\mathrm{p}=0.025$ ). The improvement was maintained from the first injection to the six-months follow-up for both groups.

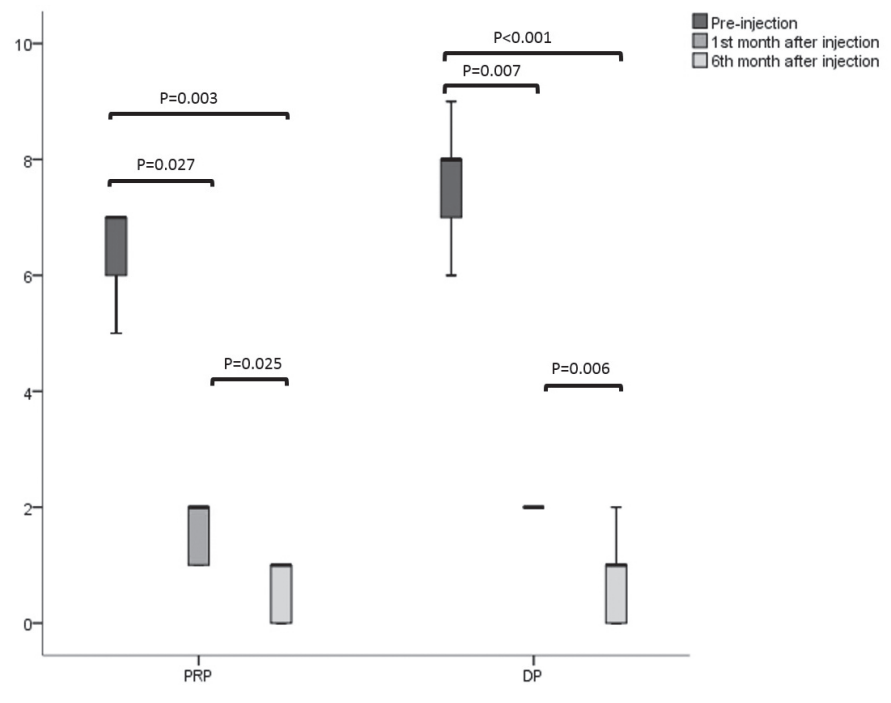

FIGURE 1. The box plots showing the initial and follow-up Visual Analogue Scale (VAS) scores of dextrose prolotherapy (DP) and platelet-rich plasma (PRP) group

\section{Nirschl Pain Phase Scale Scores}

Both groups showed significant improvements in the NPPS scores at all follow-ups. At the 1st month, functional improvements were observed according to NPPS scores within each group compared to the initial scores (Table 1). There were no significant differences between groups regarding NPPS scores at pre-injection, 1st month and 6th month of follow-up $(\mathrm{p}=0.6, \mathrm{p}=0.9$, and $\mathrm{p}=0.9$ respectively) (Figure 2). In the DP group, the median NPPS scores were 5 (min: 3 , max: 7 ) at pre-injection, 2 (min: 1, max: 2 ) at first month and 1 (min: 0 , max: 1 ) at sixth month, and the decrease of VAS through time points was statistically significant (overall $\mathrm{p}<0.001$, from pre-injection to 1 st month $\mathrm{p}=0.007$, from 1 st month to 6 th month $\mathrm{p}=0.003$ ). In the PRP group; median NPPS scores were 4.5 (min: 3 , max: 6) at pre-injection, 1.5 (min: 1, max: 5) at first month and 0.5 (min: 0, max: 5) at sixth month, and the decrease of VAS through time points was statistically significant (overall $p=0.007$, from pre-injection to 1 st month $p=0.039$, from 1 st month to 6th month $\mathrm{p}=0.025$ ). The improvement was maintained from the first injection to 6 months' follow-up for both groups. 


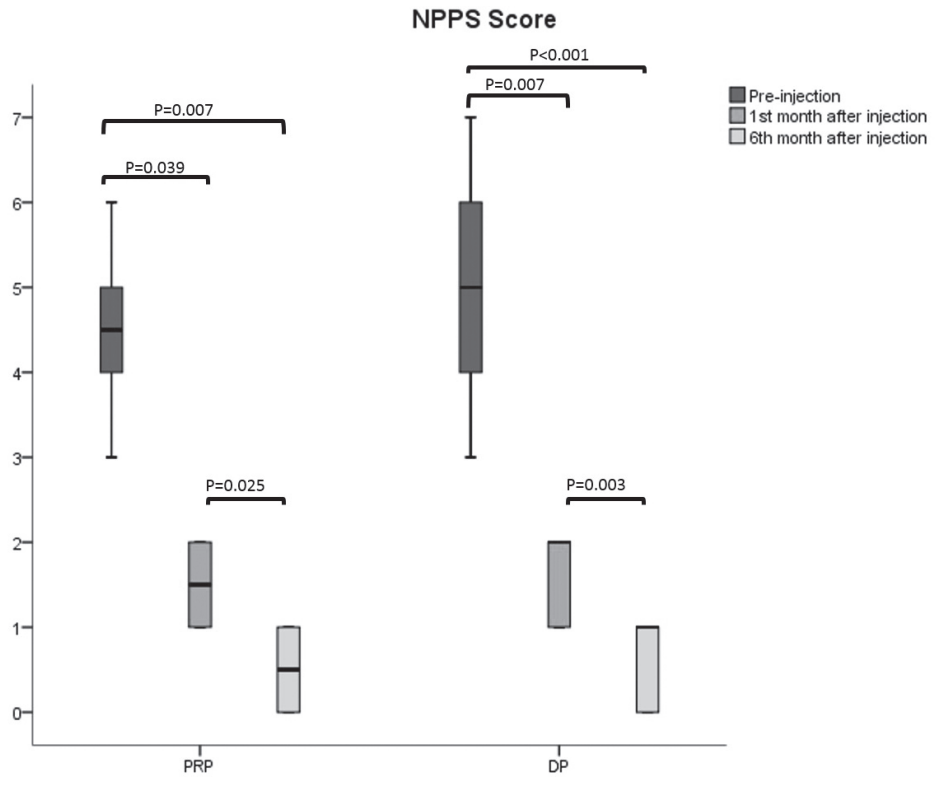

FIGURE 2. The box plots showing the initial and follow-up Nirschl Pain Phase Scale (NPPS) scores of dextrose prolotherapy (DP) and platelet-rich plasma (PRP) groups

\section{Discussion}

Pain and function changes in elite soccer players diagnosed with longstanding groin pain were assessed before and after DP and PRP injection in this study. The results of our study demonstrated the beneficial effects of both DP and PRP injection therapies on both function and pain in patients with longstanding groin pain. A few studies have proposed the favourable effects of both DP and PRP injection in groin pain (Scholten et al., 2015; Topol \& Reeves, 2008). Nevertheless, to the best of our knowledge, the effectiveness of DP and PRP on decreasing pain and improving function in groin pain was not compared. We were able to compare pain and function changes before and after DP and PRP injections.

Difference between DP and PRP injection was not significant. Both VAS and NPPS scores decreased at one and six months after DP and PRP injections. Improvement in pain and function was recorded after a month of treatment, and this effect lasted up to six months.

In an animal study that investigated the mechanical effects of prolotherapy on stretch-injured ligaments, Jensen et al. (2008) found that DP increased the cross-sectional area of medial collateral ligaments compared with saline-injected and uninjured controls; however, DP injections did not alter other measured properties in their model. Apart from the animal study, DP has been used mostly in knee osteoarthritis studies. Our findings were in line with previous DP studies that reported knee pain and functional score improvement (Rabago et al., 2013; Eslamian \& Amouzandeh, 2015). A study on lateral epicondylosis with DP reported a decrease in pain and improved grip strength compared to controls (Scarpone et al., 2008). Another study presented the benefit of DP on patients with painful knees, shoulders and lateral elbows (Lyftogt, 2007). A patient with chronic shoulder pain was treated at three sessions by DP, and they reported a $90 \%$ reduction of pain and complete restoration of normal activity (Seenauth, Inouye, \& Langland, 2018). There was only a case series using DP for the treatment of groin pain (Topol et al., 2008). Researchers treated 75 elite athletes who were suffering from groin pain with DP injections, which consisted of monthly injections of $12.5 \%$ dextrose with $0.5 \%$ lidocaine in abdominal and adductor attachments on the pubis. Similar to our results, DP therapy yielded substantial reductions in VAS and NPPS scores (Topol et al., 2008).

VAS and NPPS scores also decreased in the PRP injected groin pain patients in our study. A decrease in pain and improvement in function was recorded in one and six months after injections. Use of PRP has recently been becoming widespread as it also delivers mediators that enhance tissue healing (Kon et al., 2011). Laboratory evidence suggests that PRP may have a positive effect on tendon healing-related processes as neo-vascularization is important for regeneration (Alsousou, Thompson, Hulley, Noble, \& Willett, 2009). Clinical studies on PRP demonstrated promising results for the treatment of tendinopathies over the past few years (Gosens, Peerbooms, Van Laar, \& Den Oudsten, 2011; Peerbooms, Sluimer, Bruijn, \& Gosens, 2010). Our findings were in line with these studies. Tan et al. (2016) reported improvement in VAS and Mayo elbow scores of 56 patients who were suffering from refractory lateral epicondylitis after PRP injection. In 2006, Mishra et al. (2006) suggested that concentrated growth factors in PRP could be used to initiate a healing response in a damaged tendon. In their study evaluating the effect of PRP injection in patients with chronic elbow tendinosis, it was found that the PRP group had better results than the control group (Mishra \& Pavelko, 2006). The beneficial effects of PRP have also been demonstrated on plantar fasciitis (Kim \& Lee, 2014). Our 
findings for longstanding groin pain were in line with previous DP studies. Despite so many studies in different clinical tables using PRP injection, there are limited studies on PRP applied for groin pain (Scholten et al., 2015; St-Onge, MacIntyre, \& Galea, 2015). In a case report, Scholten et al. (2015) used ultrasound-guided needle tenotomy and PRP injection in the treatment of a male elite lacrosse player with athletic pubalgia, and they found that improved pain and return to full pain-free plays at the previous level of intensity eight weeks after the injection. In another case report, St-Onge et al. (2015) used two PRP injections to a tear in the rectus abdominis of a male hockey player. They found that the patient returned to his prior level of performance in 3.5 weeks (St-Onge et al., 2015). Our findings related to PRP injection were consistent with previous PRP studies reporting improvement in pain and functional score.

PRP is a treatment that requires sampling and condensing thrombocytes from the patient and spending time for the centrifuge process. Furthermore, this method needs valid and reliable blood processing consumables and a centrifuge. The variable properties of platelet preparation vary slightly between companies, depending on several factors (Scholten et al., 2015). DP, in contrast, is less costly and an easier option. There are currently no published studies comparing DP and PRP treatments for longstanding groin pain, and we were not able to demonstrate a difference between the two treatment options. DP is a low-cost, less time consuming, and effective longstanding groin pain treatment method as is PRP injections.

In vivo studies showed that PRP may have an anti-inflammatory effect by inhibiting the NF-KB pathway. (Utku et al., 2017) Each millilitre of PRP solution contains 1.5-2 million platelets, which represents a 5-fold increase in platelet and growth factors (Lai et al., 2015). In contrast, DP shows its effect by increasing PDGF levels. DP has been shown to be effective in the repair of connective tissue injuries due to its irritative nature (Sit et al., 2016). Both DP and PRP, although acting in different physiological ways, are useful for OP patients who did not respond to the other therapeutic options such as physical therapy and exercise therapy.

Our study has several limitations. The retrospective design is a limitation; nevertheless, reduced pain and improved function after one and six months using DP and PRP injections is a valuable finding. The small number of patients restricted our statistical methods; however, early outcomes with both methods revealed a favourable outcome. Another limitation was the absence of a placebo-controlled group. Soccer players who did not benefit from previous medical and physical therapy methods wanted to go back to their sporting activities as soon as possible, and we were not able to establish a placebo control group. The peppering technique was used in both the DP and the PRP injections. We observed that dry needling as a part of the peppering technique had therapeutic effects as it was presented in other tendon studies (Creaney, Wallace, Curtis, \& Connell, 2011; Kampa \& Connell, 2010). We do attribute the positive results we have found to the effect of treatments since the same peppering technique was used in both our treatment groups. Subsequent prospective studies with the placebo group should focus on a larger sample of participants that will provide a better understanding of the DP and PRP injections on longstanding groin pain. Despite these limitations, we have proven that DP and PRP are safe, relatively simple, and potentially effective treatment methods for the treatment of longstanding groin pain.

\section{Conclusion}

We compared time-dependent pain and function changes before and after DP and PRP injections in the treatment of longstanding groin pain. Both injections improved pain and function after a month, and this improvement sustained at six months without any reported complications. Both treatments seem to be effective for longstanding groin pain and should be considered in patients who are not responding to other conservative modalities. It should not be forgotten that DP is a cost-effective and less time-consuming treatment option for longstanding groin pain. Further prospective studies in larger patient groups are needed, in which researchers may use not only clinical measures but also radiological and biological findings as secondary outcome measures.

\section{References}

Alsousou, J., Thompson, M., Hulley, P., Noble, A., \& Willett, K. (2009). The biology of platelet-rich plasma and its application in trauma and orthopaedic surgery. The Journal of Bone and Joint Surgery. British Volume, 91-B(8), 987-996. doi: 10.1302/0301-620x.91b8.22546

Angoules, A. G. (2015). Osteitis pubis in elite athletes: Diagnostic and therapeutic approach. World Journal of Orthopedics, 6(9), 672. doi: 10.5312/wjo.v6.i9.672

Batt, M. E., McShane, J. M., \& Dillingham, M. F. (1995). Osteitis pubis in collegiate football players. Medicine and Science in Sports and Exercise, 27(5), 629-633. doi: 10.1249/00005768-199505000-00003

Choi, H., McCartney, M., \& Best, T. M. (2011, January). Treatment of osteitis pubis and osteomyelitis of the pubic symphysis in athletes: A systematic review. British Journal of Sports Medicine, Vol. 45, pp. 57-64. doi: 10.1136/bjsm.2008.050989

Creaney, L., Wallace, A., Curtis, M., \& Connell, D. (2011). Growth factor-based therapies provide additional benefit beyond physical therapy in resistant elbow tendinopathy: A prospective, single-blind, randomised trial of autologous blood injections versus platelet-rich plasma injections. British Journal of Sports Medicine, 45(12), 966-971. doi: 10.1136/bjsm.2010.082503

Cunningham, P. M., Brennan, D., O’Connell, M., MacMahon, P., O’Neill, P., \& Eustace, S. (2007). Patterns of 
Bone and Soft-Tissue Injury at the Symphysis Pubis in Soccer Players: Observations at MRI. American Journal of Roentgenology, 188(3), W291-W296. doi: 10.2214/AJR.06.0051

Dhurat, R., \& Sukesh, M. (2014). Principles and Methods of Preparation of Platelet-Rich Plasma: A Review and Author's Perspective. Journal of Cutaneous and Aesthetic Surgery, 7(4), 189. doi: 10.4103/09742077.150734

Eslamian, F., \& Amouzandeh, B. (2015). Therapeutic effects of prolotherapy with intra-articular dextrose injection in patients with moderate knee osteoarthritis: a single-arm study with 6 months follow up. Therapeutic Advances in Musculoskeletal Disease, 7(2), 35-44. doi: 10.1177/1759720X14566618

Gosens, T., Peerbooms, J. C., Van Laar, W., \& Den Oudsten, B. L. (2011). Ongoing positive effect of platelet-rich plasma versus corticosteroid injection in lateral epicondylitis: A double-blind randomized controlled trial with 2-year follow-up. American Journal of Sports Medicine, 39(6), 1200-1208. doi: $10.1177 / 0363546510397173$

Hölmich, P., Uhrskou, P., Ulnits, L., Kanstrup, I. L., Bachmann Nielsen, M., Bjerg, A. M., \& Krogsgaarda, K. (1999). Effectiveness of active physical training as treatment for long-standing adductor-related groin pain in athletes: Randomised trial. Lancet, 353(9151), 439-443. doi: 10.1016/S0140-6736(98)03340-6

Hopp, S. J., Culemann, U., Kelm, J., Pohlemann, T., \& Pizanis, A. (2013). Osteitis pubis and adductor tendinopathy in athletes: A novel arthroscopic pubic symphysis curettage and adductor reattachment. Archives of Orthopaedic and Trauma Surgery, 133(7), 1003-1009. doi: 10.1007/s00402-013-1777-7

Jardí, J., Rodas, G., Pedret, C., Til, L., Cusí, M., Malliaropoulos, N., ... Maffulli, N. (2014). Osteitis pubis: can early return to elite competition be contemplated? Translational Medicine @ UniSa, 10, 52-58. doi: 10.14273/unisa-446

Jensen, K. T., Rabago, D. P., Best, T. M., Patterson, J. J., \& Vanderby, R. (2008). Response of knee ligaments to prolotherapy in a rat injury model. American Journal of Sports Medicine, 36(7), 1347-1357. doi: $10.1177 / 0363546508314431$

Kampa, R. J., \& Connell, D. A. (2010). Treatment of tendinopathy: is there a role for autologous whole blood and platelet rich plasma injection? International Journal of Clinical Practice, 64(13), 1813-1823. doi: $10.1111 / \mathrm{j} .1742-1241.2010 .02432 . \mathrm{x}$

Kim, E., \& Lee, J. H. (2014). Autologous Platelet-Rich Plasma Versus Dextrose Prolotherapy for the Treatment of Chronic Recalcitrant Plantar Fasciitis. PM and R, 6(2), 152-158. doi: 10.1016/j.pmrj.2013.07.003

Kon, E., Mandelbaum, B., Buda, R., Filardo, G., Delcogliano, M., Timoncini, A., ... Marcacci, M. (2011). Platelet-Rich Plasma Intra-Articular Injection Versus Hyaluronic Acid Viscosupplementation as Treatments for Cartilage Pathology: From Early Degeneration to Osteoarthritis. Arthroscopy: The Journal of Arthroscopic \& Related Surgery, 27(11), 1490-1501. doi: 10.1016/J.ARTHRO.2011.05.011

Lai, L. P., Stitik, T. P., Foye, P. M., Georgy, J. S., Patibanda, V., \& Chen, B. (2015). Use of Platelet-Rich Plasma in Intra-Articular Knee Injections for Osteoarthritis: A Systematic Review. PM and R. doi: 10.1016/j. pmrj.2015.02.003

Lyftogt, J. (2007). Subcutaneous prolotherapy treatment of refractory knee, shoulder, and lateral elbow pain. Australasian Musculoskeletal Medicine, 12(2), 110-112.

McKim, K. R., Taunto, J. E., \& Kirchner, G. D. (1999). The Effectiveness of a Compression Short in the Treatment of Athletes with Osteitis Pubis. Clinical Journal of Sport Medicine, 9(2), 112. Abstracts from the Annual Symposium of the Ca.

Mishra, A., \& Pavelko, T. (2006). Treatment of chronic elbow tendinosis with buffered platelet-rich plasma. American Journal of Sports Medicine, 34(11), 1774-1778. doi: 10.1177/0363546506288850

Murphy, M., Godson, C., Cannon, S., Kato, S., Mackenzie, H. S., Martin, F., \& Brady, H. R. (1999). Suppression subtractive hybridization identifies high glucose levels as a stimulus for expression of connective tissue growth factor and other genes in human mesangial cells. The Journal of Biological Chemistry, 274(9), 5830-5834. doi: 10.1074/jbc.274.9.5830

O'Connor, F. G., Howard, T. M., Fieseler, C. M., \& Nirschl, R. P. (1997). Managing Overuse Injuries a Systematic Approach. Physician and Sportsmedicine, 25(5), 88-113. doi: 10.3810/psm.1997.05.1359

Omar, I. M., Zoga, A. C., Kavanagh, E. C., Koulouris, G., Bergin, D., Gopez, A. G., ... Meyers, W. C. (2008). Athletic Pubalgia and "Sports Hernia": Optimal MR Imaging Technique and Findings. RadioGraphics, 28(5), 1415-1438. doi: 10.1148/rg.285075217

Peerbooms, J. C., Sluimer, J., Bruijn, D. J., \& Gosens, T. (2010). Positive Effect of an Autologous Platelet Concentrate in Lateral Epicondylitis in a Double-Blind Randomized Controlled Trial. The American Journal of Sports Medicine, 38(2), 255-262. doi: 10.1177/0363546509355445

Rabago, D., Patterson, J. J., Mundt, M., Kijowski, R., Grettie, J., Segal, N. A., \& Zgierska, A. (2013). Dextrose Prolotherapy for Knee Osteoarthritis: A Randomized Controlled Trial. The Annals of Family Medicine, 11(3), 229-237. doi: 10.1370/afm.1504

Rodriguez, C., Miguel, A., Lima, H., \& Heinrichs, K. (2001). Osteitis Pubis Syndrome in the Professional Soccer Athlete: A Case Report. Journal of Athletic Training, 36(4), 437.

Scarpone, M., Rabago, D. P., Zgierska, A., Arbogast, G., \& Snell, E. (2008a). The efficacy of prolotherapy for lateral epicondylosis: A pilot study. Clinical Journal of Sport Medicine, 18(3), 248-254. doi: 10.1097/ JSM.0b013e318170fc87

Scarpone, M., Rabago, D. P., Zgierska, A., Arbogast, G., \& Snell, E. (2008b). The efficacy of prolotherapy for lateral epicondylosis: A pilot study. Clinical Journal of Sport Medicine, 18(3), 248-254. doi: 10.1097/ 
JSM.0b013e318170fc87

Scholten, P. M., Massimi, S., Dahmen, N., Diamond, J., \& Wyss, J. (2015). Successful treatment of athletic pubalgia in a lacrosse player with ultrasound-guided needle tenotomy and platelet-rich plasma injection: A case report. $P M$ and $R, 7(1), 79-83$. doi: 10.1016/j.pmrj.2014.08.943

Seenauth, C., Inouye, V., \& Langland, J. O. (2018). Dextrose Prolotherapy for Chronic Shoulder Pain: A Case Report. Alternative Therapies in Health and Medicine, 24(1), 56-60.

Sit, R. W. S., Chung, V. C. H., Reeves, K. D., Rabago, D., Chan, K. K. W., Chan, D. C. C., .. Wong, S. Y. S. (2016). Hypertonic dextrose injections (prolotherapy) in the treatment of symptomatic knee osteoarthritis: A systematic review and meta-analysis. Scientific Reports. doi: 10.1038/srep25247

St-Onge, E., MacIntyre, I. G., \& Galea, A. M. (2015). Multidisciplinary approach to non-surgical management of inguinal disruption in a professional hockey player treated with platelet-rich plasma, manual therapy and exercise: a case report. The Journal of the Canadian Chiropractic Association, 59(4), 390-397.

Tan, X. xiang, Ju, H. yang, Yan, W., Jiang, H. jiang, Su, J. ping, Dong, H. jun, ... Zou, D. bao. (2016). Autologous platelet lysate local injections for the treatment of refractory lateral epicondylitis. Journal of Orthopaedic Surgery and Research, 11(1), 2-7. doi: 10.1186/s13018-016-0349-2

Topol, G. A., \& Reeves, K. D. (2008). Regenerative injection of elite athletes with career-altering chronic groin pain who fail conservative treatment: A consecutive case series. American Journal of Physical Medicine and Rehabilitation, 87(11), 890-902. doi: 10.1097/PHM.0b013e31818377b6

Topol, G. A., Reeves, K. D., \& Hassanein, K. M. (2005). Efficacy of dextrose prolotherapy in elite male kickingsport athletes with chronic groin pain. Archives of Physical Medicine and Rehabilitation, 86(4), 697-702. doi: 10.1016/j.apmr.2004.10.007

Utku, B., Dönmez, G., Erişgen, G., Akin, Ş., Demirel, H. A., Korkusuz, F., \& Doral, M. N. (2017). Meloxicam and diclofenac do not change VEGF and PDGF-AB serum levels of platelet-rich plasma. Turkish Journal of Medical Sciences. doi: 10.3906/sag-1602-60

Verrall, G. M., Slavotinek, J. P., Barnes, P. G., \& Fon, G. T. (2005). Description of pain provocation tests used for the diagnosis of sports-related chronic groin pain: Relationship of tests to defined clinical (pain and tenderness) and MRI (pubic bone marrow oedema) criteria. Scandinavian Journal of Medicine and Science in Sports, 15(1), 36-42. doi: 10.1111/j.1600-0838.2004.00380.x

Watkin, N. A., Gallegos, C. R., Moisey, C. U., \& Charlton, C. A. C. (1995). Osteitis pubis: A case of successful treatment with anticoagulants. Acta Orthopaedica, 66(6), 569-570. doi: 10.3109/17453679509002317 